\title{
NEN - the role of somatostatin receptor scintigraphy in clinical setting
}

\author{
Marta Opalinska1, Alicja Hubalewska-Dydejczyk², Anna Sowa-Staszczak ${ }^{1}$, Agnieszka Stefanska² \\ ${ }^{1}$ Nuclear Medicine Unit, Endocrinology Department, University Hospital, Krakow, Poland \\ ${ }^{2}$ Chair and Department of Endocrinology, Jagiellonian University, Medical College, Krakow, Poland
}

[Received 8 VII 2016; Accepted 10 VII 2016]

\begin{abstract}
Detection of neuroendocrine neoplasms (NENs) and monitoring of their response to therapy is still challenging due to huge heterogeneity of that group of tumors. Actually, NENs visualization is mainly based on molecular imaging while in the past it was relied on less effective structural imaging including CT and MRI. Molecular imaging techniques in combination with structural imaging (hybrid imaging), especially in patients with well-differentiated NENs, in addition to morphological provide the functional information about tumor which benefits in a more accurate patient management, including more sensitive visualization of primary tumors, more precise staging and better therapy follow-up.

Overexpression of somatostatin receptors (SSTR) on NENs' cell membrane was a basis for development of somatostatin receptor scintigraphy (SRS) using single photon emission tomography SPECT, which is today a well-established standard in molecular imaging of NENs, and further imaging improvement in the field of positron emission tomography (PET). Use of hybrid imaging (SPECT/CT, PET/CT) increased sensitivity of examination, mainly resulting in better detection of small lesions. Generally, somatostatin receptor imaging with PET/CT is an emerging technique, although still with limited access, but due to several advantages over SSTR SPECT/CT, should be used if available. It is worth mentioning, that both SSTR PET/CT and SSTR SPECT/CT have some limitations, such as relatively low detection rate of benign insulinomas, poorly differentiated GEP-NETs and liver metastases. For that reason further improvement of NETs imaging is necessary. The most promising new tracers' families are based on SSTR antagonists, ${ }^{64} \mathrm{Cu}$-radiolabeled ligands and glucagon-like peptide-1 receptor (GLP-1R) imaging. Finally, in case of poor-differentiated neuroendocrine cancers ${ }^{18} \mathrm{~F}-\mathrm{FDG}$ PET/CT may be beneficial in comparison with molecular imaging based on somatostatin receptor modalities.
\end{abstract}

KEY words: NEN, NET imaging, octreotide, SRS, SRI, ${ }^{99 m T C-E D D A / H Y N I C-T O C, ~}{ }^{68} \mathrm{Ga}-\mathrm{PET} / \mathrm{CT}$

Nucl Med Rev 2016; 19, 2: 118-125

\section{Introduction}

Neuroendocrine neoplasms (NENs) originate from neuroendocrine (enterochromaffin) cells located in neuroendocrine tissue which are dispersed throughout the body, although are usually located in endocrine glands and in gastrointestinal and bronchopulmonary systems.

Most of NETs are sporadic but about $20 \%$ of NENs occur in the context of a genetic syndrome like multiple endocrine neoplasia types 1 and 2, and von Hippel-Lindau and neurofibromatosis type 1 and pheohromocytoma/paraganglioma syndromes.

Neuroendocrine neoplasm group cover GEP-NETs (gastroenteropancreatic neuroendocrine tumors) and lung NENs as well as other neoplasms originating from a neurocrine cells such as pheochromocytoma, paraganglioma, neuroblastoma and medullary thyroid cancer [1].

Correspondence to: Opalinska Marta, MD, PhD

Nuclear Medicine Unit, Endocrinology Department,

University Hospital, Krakow

ul. Kopernika 17, 31-501 Krakow, Poland

Tel.: (+ 48 12) 424 7510; fax: (+48 12) 4247399

E-mail: mkal@vp.pl, tel. 124247510
NENs are characterized by expression of general neuroendocrine tumor markers such as chromogranin A ( $\mathrm{CgA})$ and synaptophysin. In addition they have an ability to produce peptides, but clinically they can be either asymptomatic or symptomatic due to excess hormone secretion. It includes functioning carcinoid tumors and a variety of other functioning NENs arising mainly from the pancreas producing different hormones. Their names reflect clinical syndromes such as insulinoma, glucagonoma, gastrinoma, VIPoma, although the term carcinoid syndrome is used for tumors producing serotonin. The clinical consequences of the hormone hypersecretion may be significant (especially in case of insulinoma and carcinoid syndrome) but generally total prognosis depends, in majority of patients, on the grade and stage of the tumor. The last WHO 2010 classification of NENs divides them by mitotic or Ki67 index into well differentiated tumors: NET G1 ( $\mathrm{Ml}<2$, Ki-67 $\leq 2 \%$ ) and NET G2 (MI 3-20, Ki-67 3-20\%) and poor differentiated cancers: neuroendocrine cancer (NEC) ( $M I>20 \%, \mathrm{Ki}-$ $67>20 \%$ ) and mixed adenoneuroendocrine cancers (MANEC) [2] . Recently, there is proposal of a G3 tumors division in accordance to their proliferation index into the G3a neuroendocrine tumors with Ki-67 20-55\% and G3b neuroendocrine cancers (NEC) with Ki$67>50-55 \%$. This suggestion arises from the observation of some 
Table 1. World Health Organization (WHO) 2010 Classification with modification

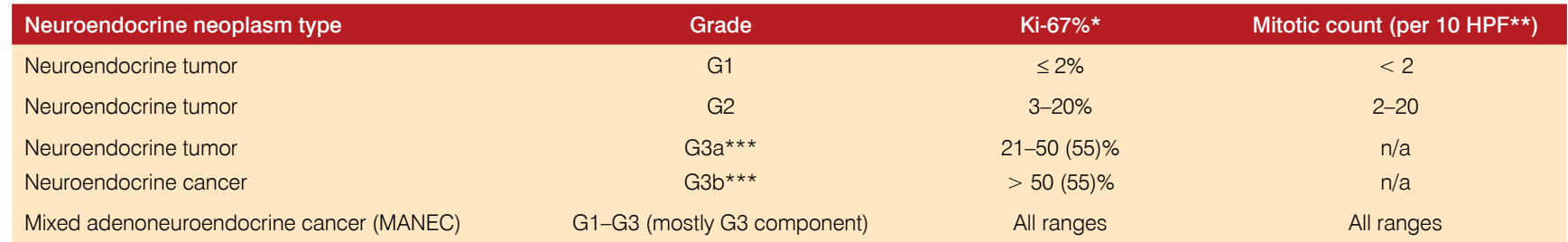

*Ki-67 index: \% of 500-2000 cells in "hot spot areas" stained positive for MIB-1 antibody; **10 HPF: high power field = $2 \mathrm{~mm}^{2}$, based on measurement in at least 50 HPFs in hot spot areas; *** proposed G3 tumor division in accordance with proliferation index; n/a - non available

Table 2. Somatostatin analog affinity profiles for human SSTRs

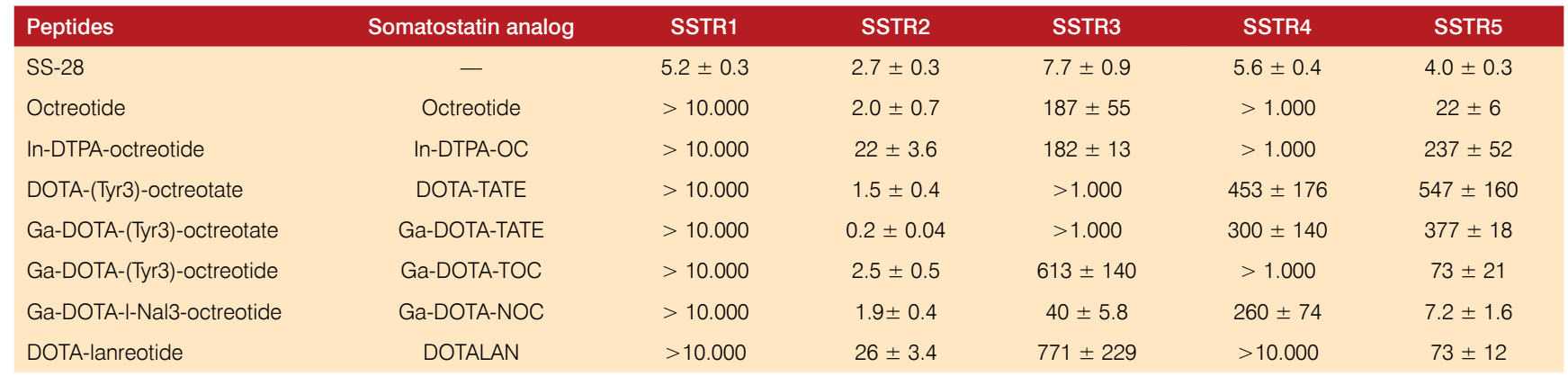

All values are $\mathrm{IC}_{50} \pm \mathrm{SEM}$ in $\mathrm{nmol} / \mathrm{l}$

differences in biology of G3 NENs. In case of G3a tumors with significant SSTR expression seen in SRI (somatostatin receptor imagining in SPECT/CT or PET/CT) there is probably a place for an attempt of therapy with "cold" somatostatin analogs (SSA) and peptide receptor radionuclide therapy (PRRT). In case of G3b cancers rather classical oncology management is suggested (Table 1).

The characteristic pattern of NENs is the overexpression of specific receptors on their cell surface, which can be visualized by molecular imaging techniques.

In this review we summarize the currently available nuclear medicine methods of NEN imaging based on visualization of somatostatin receptor.

\section{Role of somatostatin receptors in molecular imaging}

NENs imagining is challenging mainly due to varied place of tumors appearance and cellular heterogeneity starting from benign lesions up to highly aggressive cancers. In many cases the structural imaging techniques, like ultrasound, computed tomography (CT) or magnetic resonance (MR) have suboptimal diagnostic sensitivity making especially diagnosis of the limited disease ineffective. Therefore actual guidelines emphasize the importance of functional imaging for evaluating the extent of NENs. Molecular imaging techniques also in combination with CT or MRI (hybrid imaging) in case of well differentiated neoplasm are beneficial in comparison with structural imaging especially in localization of primary tumors and disease staging.

Neuroendocrine cells signal to other cells controlling many physiological processes by secretion of peptides binding to stimulatory or inhibitory receptors on targeted cells. The most important among them are somatostatin receptors having in human 5 subtypes, SSTR1-5, often coexisting in the same cells with different expression on normal and cancer cells [3].
The distribution of SSTR in NENs differs, depending on the type of the tumor and degree of its differentiation. Among them SSTR2 and SSTR5 are usually overexpressed on NENs cells, while normal tissue express mainly SSTR3 and 5.

According to literature, the pancreatic NENs such as gastrinomas express SSTR2 in about 100\%, SSTR5 in 35\%, SSTR3 in $20 \%$ and SSTR1 in 10\%, insulinomas express SSTR2 in 70\%, SSTR1 in 60\%, SSTR3 in 35\% and SSTR4 in 3\% and jejunoileal express SSTR2 in about 95\%, SSTR1 in 50\%, SSTR5 in 48\% and SSTR3 in 15\%, SSTR 4 in 3\% [4]. Other currently clinically useful receptors in imaging of NEN are glucagon-like peptide-1 receptor (GLP-1R) and insulinotropic peptide receptor (GIPR) [3].

Naturally occurring somatostatin (SST) is a peptide having 2 active forms, containing 14 and 28 amino acids, both binding to all five subtypes of SSTR. It has a very low metabolic stability in vivo ( $<2$ minutes) forcing development of synthetic somatostatin analogues needed for imaging and therapy. By now several types of them have been produced, characterized by increased resistance to peptidases and having varying affinity to the SSTR subtypes [5] (Figures 1, 2). SST analogs affinity profiles for human SSTR are summarized in Table 2. SSTR subtypes expression may be different in the primary tumor and in metastases may also change during a treatment causing diagnostic confusion and change of patient prognosis.

Physiological uptake of somatostatin analogs occurs in the pituitary, salivary glands, thyroid, liver, spleen, adrenals, pancreas, kidneys, ureters, urinary and gall bladder. The greatest confusion usually concerns adrenal glands, pancreas and spleen where physiological uptake can influence diagnosis of NENs [6] and the liver where physiological high uptake of the tracer may influence metastases recognition. In addition, it is worth to remember that tumors other than NENs including small cell lung cancer, breast and ovary cancers, Hodgkin and non-Hodgkin lymphomas, gliomas and meningiomas can overexpress SSTR in different pattern [7] causing that all of them can potentially be visualized by SRI. Moreover, 


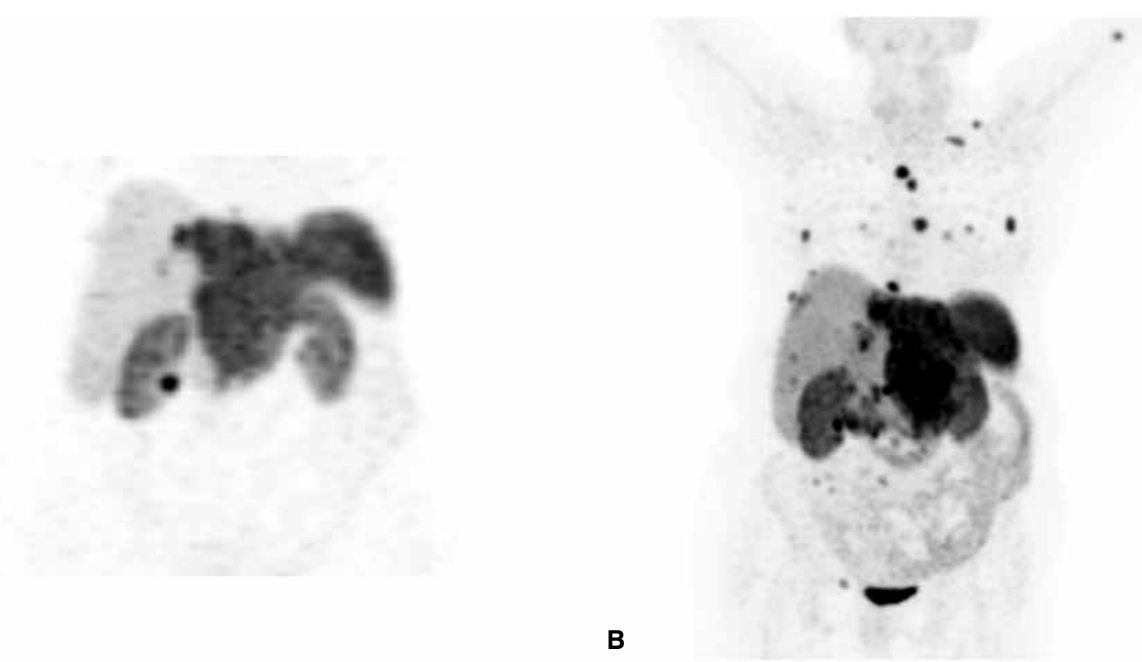

Figure 1. 77 year-old-women with liver NEN (NET G2 according to WHO 2010 classification). A. ${ }^{99 m T C-H Y N I C-T O C ~ S P E C T / C T ~ o f ~ a b d o m e n ; ~ B . ~}$ follow-up ${ }^{68} \mathrm{Ga}$-DOTA-TATE PET/CT. True or false NEN progression? Are all of the extra "hot spots" a sign of the disease progression or are only the effects of use of different SSA or more sensitive method of visualization?

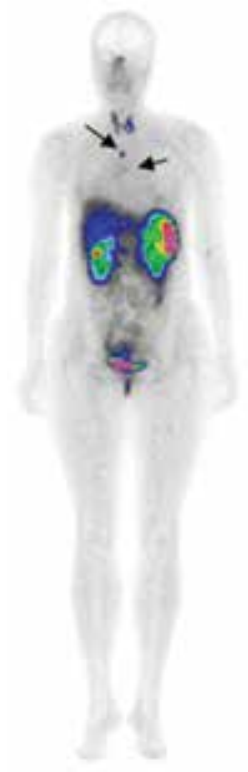

A
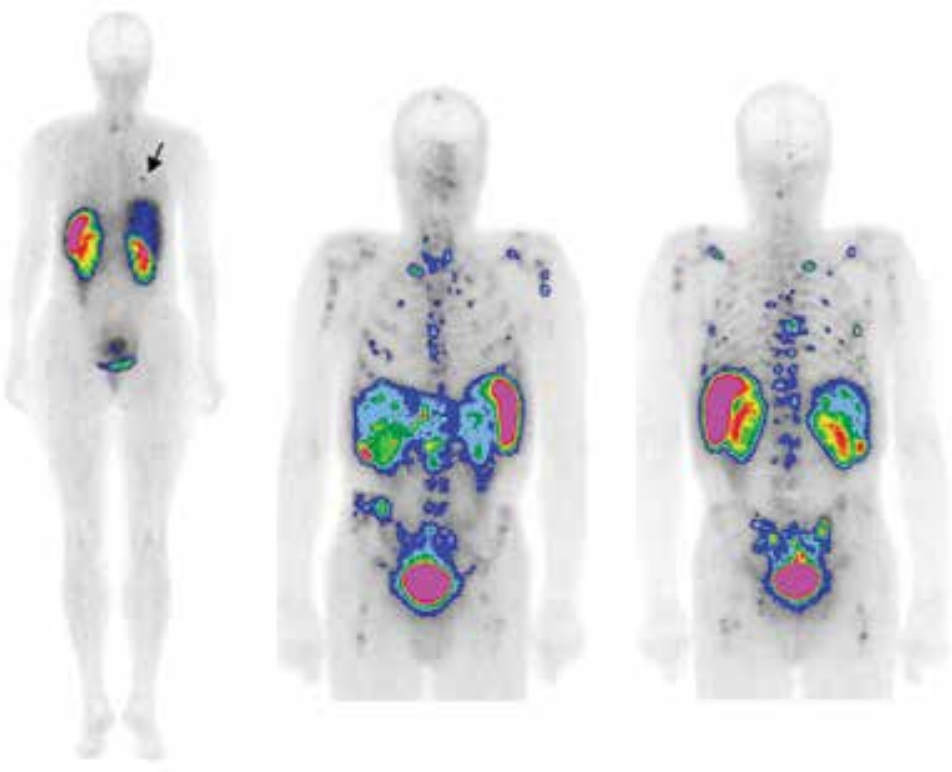

B

Figure 2. ${ }^{99 m}$ TC-HYNIC-TATE scintigraphy. A. 46-year-old women with bronchial NEN having expression of only SSTR 3 (obtained in histopathology); B. 56-year-old man with duodenal NEN having expression of all subtypes of SSTR (obtained in histopathology: high 2A, good 1, 2B, moderate 3,5 )

some somatostatin analogs accumulation may occur in benign lesions like respiratory tract infection, probably due to local lymphocytes activation, in the infection of joints including rheumatoid arthritis, Wegener's granuloma, tuberculosis, sarcoidosis, in healing scars, aeries underwent radiotherapy [8] or due to osteoclastic activity in degenerative bone diseases, fractures, fibrous dysplasia or vertebral hemangioma [6].

\section{Somatostatin analogs in molecular imaging}

The role of molecular imaging in diagnosis, staging and follow-up of NETs increases with concomitant development of hybrid imaging and new tracers' synthesis including use of positron emitters.
First radiopharmaceutical used in SRS was ${ }^{111}$ In-diethylenetriaminepentaacetic acid-D-Phe ${ }^{1}$-octreotide ( ${ }^{111}$ In-DTPA-octreotide) which became an integral part of the management of patients with NENs. Later, alternative somatostatin receptor agonists, labeled with the ${ }^{99 \mathrm{~m}} \mathrm{Tc}$ obtained from ${ }^{99} \mathrm{Mo} / 9{ }^{99 \mathrm{~m}} \mathrm{Tc}$ radionuclide generators were available and were extensively used in the clinical practice, such as ${ }^{99 m}$ Tc-EDDA-hydrazinonicotinyl-Tyr ${ }^{3}$-octreotate ( $\left.{ }^{99 m} \mathrm{TC}-\mathrm{HYNIC}-\mathrm{TATE}\right)$ [9] and ${ }^{99 m}$ Tc-EDDA-hydrazinonicotinyl-Tyr ${ }^{3}$-octreotide (99mTc-EDDA/ /HYNIC-TOC) [10]. Both of these tracers seem to have similar NENs sensitivity [11]. Use of ${ }^{99 m} \mathrm{Tc}$ instead of ${ }^{111} \mathrm{In}$ has lots of advantages like lower costs of examination, earlier image acquisition and wider availability with most studies comparable or higher sensitivities to ${ }^{111}$ In-DTPA-octreotide $[12,13]$. 
The next generation of labeled SSA are tracers for PET. Currently three major, clinically useful, tracers for PET/CT imaging radiolabeled with ${ }^{68} \mathrm{Ge} /{ }^{68} \mathrm{G}$ a radionuclide generator obtained ${ }^{68} \mathrm{Ga}$ are available: ${ }^{68} \mathrm{Ga}-\mathrm{DOTA}$-Phe ${ }^{1}$-Tyr ${ }^{3}$-Octreotide (DOTATOC), ${ }^{68} \mathrm{Ga}$-DOTA-Nal3-Octreotide (DOTANOC), and ${ }^{68} \mathrm{Ga}$-DOTA-Tyr ${ }^{3}$-Octreotate (DOTATATE) [10]. These tracers present some differences in pharmacokinetics but more importantly, their affinity to SSTR subtypes varies [8]. All of them can bind to SSTR2 while ${ }^{68} \mathrm{Ga}$-DOTANOC also has affinity for SSTR 3 and 5 and ${ }^{68}$ Ga-DOTATOC for SSTR 5 [14].

\section{Clinical utility of SSTR imaging}

Methods of SSTR visualization have well established position in NENs imaging. Its utility is especially connected with GEP-NETs and lung carcinoid visualization. The majority of these tumors express high number of SSTRs, present on both primary and metastatic sites [1]. In that cases SSTR imaging facilitates patients management including staging, detection of new sites of disease in patients with treated NETs (restaging), diagnosis of patients with suspicion of NEN based on biochemical or clinical findings, localization of primary tumor in case of initially disseminated disease, selection of candidates for cold SSA or peptide receptor radionuclide therapy PRRT [15]. Finally, those methods are useful in monitoring of response to the therapy. In patients with metastatic or inoperable NENs that have little or no uptake of radiolabeled SSA, the likelihood of response to long-acting SSA is significantly reduced and these patients are also unsuitable for PRRT.

Sensitivity of different imaging modalities varies for specific NENs. SRS specificity for detection of primary GEP-NETs is $86-95 \%$ and is higher than for location of pancreatic gastrin/VIP/somatostatin-secreting NETs (75\%) and insulinomas (50-60\%) [16]. In various studies sensitivity of ${ }^{68} \mathrm{Ga}$-DOTA-peptides in PET/CT detection of NETs has been proven higher than for SRS [17-19]. For primary NENs its sensitivity is estimated at $78-93 \%$ and is highest for pancreatic NENs (86-100\%) except insulinomas in which case the sensitivity is in general lower starting from $25 \%$ in case of ${ }^{68} \mathrm{Ga}$-DOTANOC [20] up to $87 \%$ in case of DOTATOC [21]. Similar effectiveness for ${ }^{68} \mathrm{Ga}$-DOTA-peptides was established in PET/CT in patients with clinical/biochemical/radiological suspicion of NENs [22].

Among gastric NENs the somatostatin receptor imaging can be useful in type 2 and 3 of NENs as a part of the overall staging and may facilitate choosing of therapy. Somatostatin receptor imaging is seldom useful for type 1 gastric NENs which are usually indolent and require mainly gastroscopic supervision [23].

According to literature, SRI in combination with CT may be considered in patients with appendix NEN, while resection of NEN is considered as not complete or when distant metastatic disease is suspected [24].

For jejunum and ileum, NENs SRI has sensitivities of approximately $90 \%$ for primary tumors with or without nodal involvement and $>95 \%$ for liver metastases. That indicate SRI as an important tool for staging and follow-up in patients with jejunum and ileum NENs [25]. For that group of NENs ${ }^{68} \mathrm{Ga}-\mathrm{DOTA}$-peptides PET/CT was confirmed to be more useful in comparison with ${ }^{111}$ In-DTPA octreotide SPECT/CT in searching for a primary tumor in patients with unknown or suspected disease.

Due to the high sensitivity of SRI in detection of pancreatic NENs, especially in PET/CT techniques, use of ${ }^{68} \mathrm{Ga}$-DOTA-pep- tides is now the method of choice to fully stage and localize the extent of disease in patients with non-insulinoma pancreatic NETs [26].

In case of colorectal NENs, tumor size and its depth predict node metastasis. Intramucosal tumors $<1 \mathrm{~cm}$ have a $4 \%$ risk of lymph node metastasis. Although the risk of metastasis of colorectal NENs is not zero even for small tumors, the majority of patients appear cured once full resections of small $(<10 \mathrm{~mm})$ rectal NENs with favorable biology is done. In case of tumors $>2 \mathrm{~cm}$ the risk of metastatic disease is $60 \%$. For that reasons ENETs recommend SRI, as one of visualization possibility, in patients with tumors $>2 \mathrm{~cm}$ and grade 1 and 2 or tumors $>1 \mathrm{~cm}$ grade 3 or suspicion of metastasis [27].

Lung NENs express SSTR1 and SSTR2 in 70\% and SSTR5 in $20 \%$ [28]. According to literature for typical bronchial carcinoids SRI, especially in PET/CT techniques is a sensitive tool of its visualization and has in general higher sensitivity than for atypical ones [5, 29].

Generally, well-differentiated tumors are detected in SRI with higher sensitivity than high-grade tumors which are usually seen in ${ }^{18} \mathrm{~F}-\mathrm{FDG}$ PET/CT. However somatostatin receptor scintigraphy is not routinely indicated in G3 tumors, but may be considered in tumors with proliferative indexes in the low range of G3 (Ki$67<55 \%$ ) [30].

In case of metastatic disease from NENs, the metastases are predominantly found in the liver, lymph nodes and/or bones changing grading of the disease. Use in comparable clinical trials more sensitive imaging methods such as ${ }^{68} \mathrm{Ga}-\mathrm{DOTA}$ peptides PET/CT shows that real number of metastases especially in bones is higher than estimated by conventional radiological techniques [31].

According to literature, ${ }^{68} \mathrm{Ga}$-DOTA-peptides have been shown to change the management (surgical, medical, staging) in comparison with structural imagining (CT, MRI) and even with SRS. The diagnostic accuracy of SSTR PET/CT in patients with known NEN is higher than CT or MRI [32]. PET/CT results change clinical management in $38 \%-60 \%$ of the examined patients. In patients with negative or equivocal ${ }^{111}$ In-DTPA-octreotide findings, ${ }^{68} \mathrm{Ga}$-DOTATATE PET identified significantly more lesions than ${ }^{111}$ In-DTPA-octreotide scintigraphy [17]. Simultaneously done SRS with ${ }^{99 m}$ TC-HYNIC-TOC or ${ }^{111}$ In-DOTA-TOC and compared with ${ }^{68} \mathrm{Ga}-\mathrm{DOTA}-\mathrm{TOC}$ PET showed higher sensitivity of PET/CT which provided additional valuable clinical information in $14.3 \%$ of investigated patients, mainly reviling non-seen in SRS bone metastases [18]. The consequence of above findings cause ${ }^{68} \mathrm{Ga}$-DOTA-peptides PET/CT should be the method of choice in management of well differentiated NENs (especially GEP-NETs tumors excluding patient with suspicion of insulinoma) [15].

The high expression of SSTR enables also SRI imaging of other neuroendocrine tumors being in some cases useful in staging, restaging and follow-up of pheochromocytomas, paragangliomas, neuroblastomas or medulloblastomas [28].

\section{Insulinoma visualization}

Due to relatively low sensitivity of SRI, especially in case of benign, insulinomas there is a need for new tracer synthesis dedicated for insulinoma detection. Strong overexpression of glucagonlike peptide-1 (GLP-1) receptors in human insulinoma provides it as an attractive target for imaging.

By now, clinical trials have demonstrated usefulness of GLP-1 receptor in SPECT/CT imagining using (Lys ${ }^{40}$ (Ahx (6-ami- 


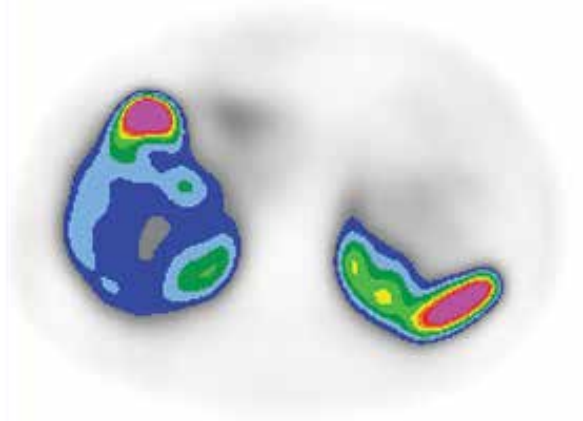

A

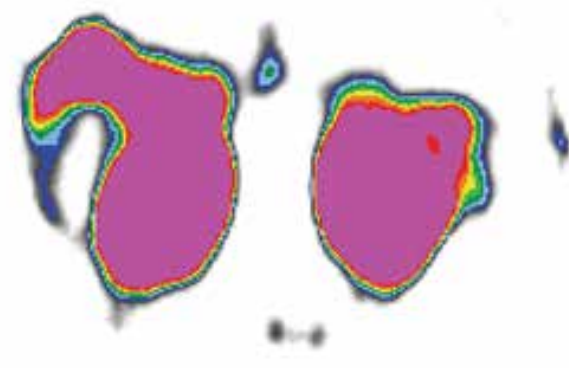

Figure 3. 62-year-old women with MEN1 syndrome with suspicion of insulinoma recurrence due to clinical symptoms. A. Negative result of ${ }^{99 m} \mathrm{mc}-$ HYNIC-TOC scintigraphy; B. clearly visible in ${ }^{99 m}$ TC-GLP-1 scintigraphy pancreatic tumor

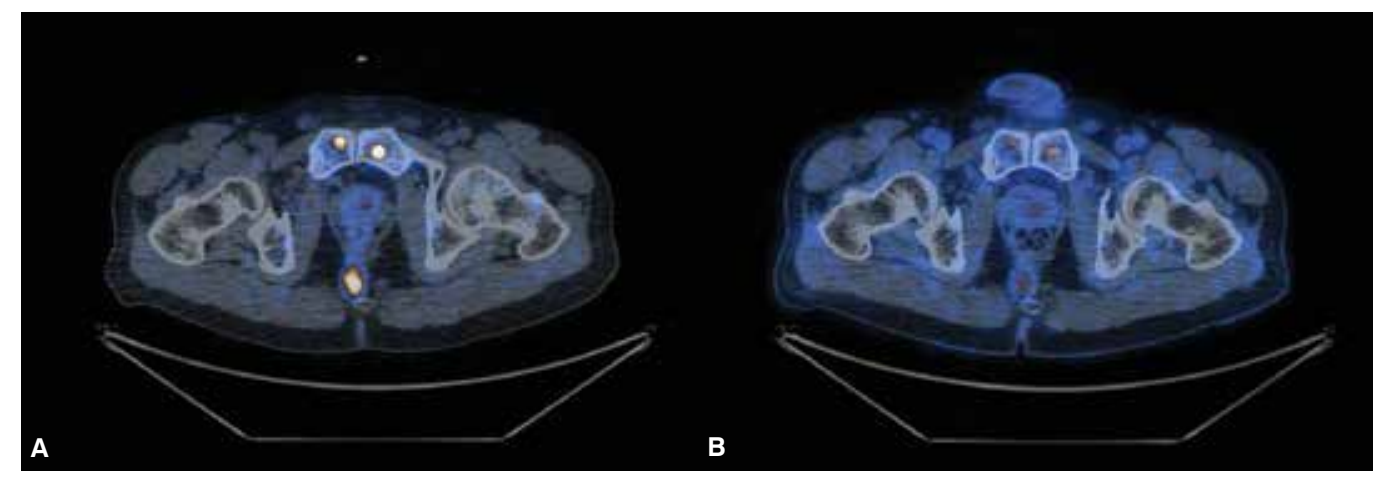

Figure 4. Comparison of high uptake of ${ }^{68} \mathrm{Ga}$-DOTATATE (A) in the rectum and metastases to pubic bone with moderate uptake of ${ }^{18} \mathrm{~F}$-FDG $(\mathrm{B})$ in PET/CT in 56-year-old men with metastatic NEN (NET G2 according to WHO 2010 classification) done to localization of primary tumor

nohexanoic acid)-DOTA- $\left.{ }^{111} \mathrm{In}\right) \mathrm{NH}_{2}$ )-exendin-4 as well as causing relatively lower radiation burden for the patient $\left(\right.$ Lys ${ }^{40}\left(\mathrm{Ahx}-\mathrm{DOTA}-{ }^{68} \mathrm{Ga}\right)$ $\mathrm{NH}_{2}$ )-exendin-4 for PET/CT and (Lys ${ }^{40}$ (Ahx-hydrazinonicotinamide (HYNIC)- ${ }^{-99 m T C)} \mathrm{NH}_{2}$ )-exendin-4 for SPECT/CT [27, 34] (Figure 3).

\section{Somatostatin receptor imaging in SPECT/CT}

SRS in SPECT/CT technique has well established position in management of NENs patients. After development of tracers based on generator obtained ${ }^{99 m} \mathrm{Tc}$, confirmation of its diagnostic sensitivity and favorable dosimetry [35] caused that value and availability of SRS for localization of primary tumor, staging, follow-up and qualification to PRRT significantly increased. Currently, for commercial use ${ }^{99 m}$ TC-EDDA/HYNIC-TOC (Tektrotyd, Poland) is available in 19 European countries and is indicated for use in adults for indication mention above. Relatively low cost of that tracers and its wide accessibility cause that SRS is still a very useful tool in NEN patients management especially in areas with limited access to PET/CT scanners.

\section{Somatostatin receptor imaging in PET/CT}

Since PET/CT scanners are relatively easy available there is a trend to shift NENs diagnostic from conventional SRS to PET/CT mainly because of their multifocal superiority in comparison with gamma-emitters. In case of PET/CT examination spatial resolution is much higher toward SPECT (3-6 mm versus $10-15 \mathrm{~mm}$ ), which provides mainly better visualization of small lesions. PET/CT save time because at once it is possible to scan all body not only a one region. Moreover there is commercially available generator $\left({ }^{68} \mathrm{Ge} /{ }^{68} \mathrm{Ga}\right)$ so there is no need of cyclotron use and PET/CT may be available in any Nuclear Medicine Unit. The long life of the mother radionuclide ${ }^{68} \mathrm{Ge}$ (270.8 days) allows for generator use even 12 months and the elution can be done even every 3 hours. As for as SRS discontinuation of long acting somatostatin analog before examination is recommended, although is not mandatory. This has clinical implication that a wide spectrum of ligands ( ${ }^{68} \mathrm{Ga}-\mathrm{DOTA}$-peptides) should be invited for NENs imaging. Some authors suggest that ${ }^{68} \mathrm{Ga}$-DOTANOC is more sensitive than SSTR2 specific ${ }^{68} \mathrm{Ga}$-DOTATATE [36]. However, there is currently no evidence of a clinical impact of these differences in SSTR binding affinity, and therefore no preferential use of one compound over the others is recommended [6].

\section{${ }^{18}$ F-FDG PET/CT in NENs imagining}

For a well-differentiated NENs PET scanning with the use of ${ }^{18} \mathrm{~F}-\mathrm{FDG}$ is not recommended due to low sensitivity for $\mathrm{G} 1$ and in many cases G2 tumors (95\%sensitivity of ${ }^{68} \mathrm{Ga}$-DOTATATE for GEP-NETs grade G1 and 2 versus $37 \%$ in case of ${ }^{18} \mathrm{~F}-\mathrm{FDG}$ ) [37] (Figure 4). In the group of patients with the Ki-67 > 15\% the ${ }^{18} \mathrm{~F}-\mathrm{FDG}$ PET/CT uptake was find in over $90 \%$ patients [38]. In all patients ${ }^{18} \mathrm{~F}-\mathrm{FDG} \mathrm{PET} / \mathrm{CT}$ may provide complementary information 
to SSTR imaging having prognostic value. Loss of SSTR receptors by NENs is usually connected with higher percentage of cells demonstrating the proliferation marker Ki-67, higher ${ }^{18} \mathrm{~F}-\mathrm{FDG}$ uptake and poorer prognosis. Additionally, ${ }^{18} \mathrm{~F}-\mathrm{FDG}$ PET may be considered in patients in whom radical surgery is being pursued or if clarification of equivocal findings on conventional imaging may change the therapeutic approach [30].

Some initial studies suggest correlation between ${ }^{68} \mathrm{Ga}-\mathrm{DOTA}$ -TATE or ${ }^{18} \mathrm{~F}-\mathrm{FDG}$ uptake and tumor grade on histology. Since the most aggressive NENs are often non seen in SSTR imaging and usually seen in ${ }^{18} \mathrm{~F}$-FDG PET, there is probably diagnostic future for staging patients with NENs who are negative in SSTR imaging [39].

\section{Other NENs specific tracers}

In last few years ${ }^{18} \mathrm{~F}$-DOPA (dihydroxyphenylalanine) has appeared. As a precursor of neurotransmitters, DOPA is taken up by some of neuroendocrine cells. In initial studies comparing ${ }^{18} \mathrm{~F}-\mathrm{DOPA}$ with ${ }^{68} \mathrm{Ga}$-DOTA-peptides, higher sensitivity of ${ }^{68}$ Galabeled tracers than ${ }^{18} \mathrm{~F}$-DOPA in GEP-NET localization was shown [40] as well as in detection of well-differentiated metastatic NENs [41]. Moreover, ${ }^{68} \mathrm{Ga}$-DOTANOC was more sensitive to ${ }^{18} \mathrm{~F}$-DOPA and ${ }^{123}$-MIBG [42] in case of tumors which should prefer amine precursor uptake, like pheochromocytoma, paraganglioma or medullary thyroid cancer.

Observation of a correlation between SUV max of ${ }^{18} \mathrm{~F}$-DOPA and serotonin plasma serum level may suggest some usefulness of this tracer in detection of tumors secreting serotonin, not visualized by SRI [43]. Gluc-Lys ${ }^{18} \mathrm{~F}-\mathrm{FP}-\mathrm{TOCA}$ is another SSTR targeted radiopharmaceutical with initial results suggesting comparable sensitivity and specificity of ${ }^{68} \mathrm{Ga}$-DOTATATE in detection of NENs [44]. 11 C-5-HTP (hydroxytryptophan) have shown promising results in detection of NENs, but further studies are necessary to confirm its utility [45]. Medullary thyroid cancer has an ability to take up gastrin and CCK-2 analogs which can be potential tools for diagnostic approach in that malignancy $[46,47]$.

\section{Future perspectives}

The role of SSTR imaging with radiolabeled somatostatin analogs is well established in clinic, but there is still a lot of uncertainty which needs elucidation, including selecting of the most sensitive peptides for NENs or their subgroups imagining. Use of different radionuclides and radiolabeling strategies influences peptide's SSTR affinity and changes radiopharmaceutical properties. By now, ${ }^{18} \mathrm{~F}-\mathrm{SSTR}$ tracers were synthesized but they did not succeed as much as tracers radiolabeled with for example ${ }^{64} \mathrm{Cu}$, such as ${ }^{64} \mathrm{Cu}$-DOTATATE [48] or ${ }^{64} \mathrm{Cu}$-TETATOC [49]. It is expected that tracers based on ${ }^{64} \mathrm{Cu}$ provide images with better resolution, higher sensitivity and will have favorable dosimetry allowing their therapeutic use. In the quest for ligands with a wider receptor affinity profile the ${ }^{177} \mathrm{Lu} /{ }^{68} \mathrm{Ga}-\mathrm{DOTA}$-AM3 has been developed which is a new bicyclic somatostatin-based radiopeptide with high affinity for all 5 subtypes of SSTR. The pharmacokinetic data make this peptide an excellent candidate as an imaging radiotracer [50]. Finally, investigation of other specific tracers as GLP-1R analogs for insulinomas or CCK-2 analogs for MTC may aid in NENs visualization.
The first in vitro study suggests that the use of a cocktail of 3 radioligands binding to somatostatin receptors, GLP-1 receptors, and GIP receptors would allow detecting virtually all NETs and labeling them homogeneously in vivo, representing a significant improvement for imaging and therapy in NETs [51]. Furthermore, using of somatostatin receptor antagonist, such as ${ }^{111}$ In-DOTA-BASS [52] or ${ }^{68} \mathrm{Ga}-\mathrm{NODAGA}-J R 11$ [53], may in future improve diagnostic value of SRI techniques.

\section{Conclusion}

Conventional radiological imaging modalities, including ultrasound, CT, MRI and EUS, are important tools in NEN patients' management, but in many cases especially in well-differentiated tumors are not always sufficient enough for the detection of the primary tumor, staging and evaluation of treatment response.

Molecular imaging techniques, especially with the use of SSA, increase sensitivity of NENs imaging. Introduction to NENs diagnostic hybrid imaging modalities and tracers based on SSA had a noticeable impact in NENs patient management. The developments in SRS followed by the progress in the design of PET tracers additionally facilitated localization of primary tumors, improved staging, restaging and follow-up.

It should be emphasized that using of SSTR SPECT/CT is still a valuable imaging method of NENs especially in case of limited access to PET/CT scanners.

${ }^{18} \mathrm{~F}-\mathrm{FDG} \mathrm{PET} / \mathrm{CT}$ is not recommended as first-line diagnostic option for well-differentiated NENs imaging but in some cases may deliver complementary information to SRI especially in case of more aggressive tumors influencing clinical management and having prognostic value. In insulinomas, especially in benign ones, due to low sensitivity of SRI, the use of GLP-1R analogs may be considered.

\section{References:}

1. Reubi JC, Kvols L, Krenning E. Distribution somatostatin receptors in normal and tumor tissue. Metabolism 1990; 39: 78-81

2. Oberg K, Castellano D. Current knowledge on diagnosis and staging of neuroendocrine tumors. Cancer Metastasis Rev. 2011; 30 Suppl 1: 3-7.

3. Reubi JC. Somatostatin and other peptide receptors as tools for tumor diagnosis and treatment. Neuroendocrinology 2004; 80 Suppl 1: 51-56.

4. Pepe G, Moncayo R, Bombardieri E, Chiti A. Somatostatin receptor SPECT. Eur J Nucl Med Mol Imaging 2012; 39 Suppl 1: S41-51.

5. Johnbeck CB, Knigge U, Kjær A. PET tracers for somatostatin receptor imaging of neuroendocrine tumors: current status and review of the literature. Future Oncol 2014; 10: 2259-2277.

6. Hofman MS, Lau WF, Hicks RJ. Somatostatin receptor imaging with $68 \mathrm{Ga}$ DOTATATE PET/CT: clinical utility, normal patterns, pearls, and pitfalls in interpretation. Radiographics. 2015; 35: 500-516.

7. Hofland LJ, Lamberts SW. Somatostatin analogs and receptors. Diagnostic and therapeutic applications. Cancer Treatment and Research 1997; 89: 365-382.

8. Ćwikła JB, Królicki L, Buscombe JR et al. Diagnostic imaging of neuroendocrine tumours Oncol Clin Pract 2006; 2: 18-31.

9. Hubalewska-Dydejczyk A, Fröss-Baron $\mathrm{K}$ et al. ${ }^{99 m} \mathrm{Tc}-\mathrm{EDDA} / \mathrm{HYNIC-oc-}$ treotate scintigraphy, an efficient method for the detection and staging of carcinoid tumours: results of 3 years' experience. Eur J Nucl Med Mol Imaging 2006; 33: 1123-1133. 
10. Parisella MG, Chianelli $M$, D'Alessandria $C$ et al. A. Clinical indications to the use of ${ }^{99 m}$ TC-EDDA/HYNIC-TOC to detect somatostatin receptor-positive neuroendocrine tumors. Q J Nucl Med Mol Imaging 2012; 56: 90-89.

11. Cwikla JB, Mikolajczak R, Pawlak D et al. Initial Direct Comparison of ${ }^{99 m}$ Tc-TOC and ${ }^{99 m}$ Tc-TATE in Identifying Sites of Disease in Patients with Proven GEP NETs. J Nucl Med 2008; 49: 7

12. Hicks RJ. Use of molecular targeted agents for the diagnosis, staging and therapy of neuroendocrine malignancy. Cancer Imaging 2010; 10 Spec no A: S83-91.

13. Hubalewska-Dydejczyk A, Fröss-Baron K, Mikołajczak R et al. ${ }^{99 m} T c-E D D A /$ /HYNIC-octreotate scintigraphy, an efficient method for the detection and staging of carcinoid tumours: results of 3 years' experience. Eur J Nucl Med Mol imaging 2006; 33: 1123-1133.

14. Virgolini I1, Ambrosini V, Bomanji JB et al. Procedure guidelines for PET/CT tumour imaging with 68Ga-DOTA-conjugated peptides: 68Ga-DOTA-TOC, 68Ga-DOTA-NOC, 68Ga-DOTA-TATE. Eur J Nucl Med Mol Imaging 2010; 37: 2004-2010

15. 15. Baumann T, Rottenburger C, Nicolas G, Wild D. Gastroenteropancreatic neuroendocrine tumours (GEP-NET) - Imaging and staging. Best Pract Res Clin Endocrinol Metab 2016; 30: 45-57.

16. Ramage JK, Ahmed A, Ardill $\mathrm{J}$ et al. Guidelines for the management of gastroenteropancreatic neuroendocrine (including carcinoid) tumours (NETs). Gut 2012; 61: 6-32

17. Kowalski J, Henze M, Schuhmacher J, Mäcke HR, Hofmann M, Haberkorn U. Evaluation of positron emission tomography imaging using [68Ga]-DOTA-D Phe (1)-Tyr (3)-octreotide in comparison to [111ln]-DTPAOC SPECT. First results in patients with neuroendocrine tumors. Mol Imaging Biol 2003; 5: 42-48.

18. Gabriel M, Decristoforo C, Kendler D et al. ${ }^{68} \mathrm{Ga}$-DOTA-Tyr ${ }^{3}$-octreotide PET in neuroendocrine tumors: comparison with somatostatin receptor scintigraphy and CT. J Nucl Med 2007; 48: 508-518.

19. Srirajaskanthan R, Kayani I, Quigley AM, Soh J, Caplin ME, Bomanji J. The role of ${ }^{68} \mathrm{Ga}$-DOTATATE PET in patients with neuroendocrine tumors and negative or equivocal findings on ${ }^{111}$ In-DTPA-octreotide scintigraphy. J Nucl Med 2010; 51: 875

20. Sharma P, Arora S, Karunanithi S et al. Somatostatin receptor based PET/CT

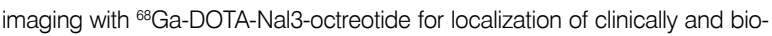
chemically suspected insulinoma. Eur J Nucl Med Mol Imaging 2016; 60: 69-76.

21. Prasad V, Sainz-Esteban A, Arsenic R et al. Role of $68 \mathrm{Ga}$ somatostatin receptor $\mathrm{PET} / \mathrm{CT}$ in the detection of endogenous hyperinsulinaemic focus: an explorative study. Q J Nucl Med Mol Imaging 2016; 60: 69-76.

22. Ambrosini $V$, Campana $D$, Nanni $C$ et al. Is Ga-DOTA-NOC PET/CT indicated in patients with clinical, biochemical or radiological suspicion of neuroendocrine tumour? Eur J Nucl Med Mol Imaging 2012; 39: 1278-1283.

23. Delle Fave G, O'Toole D, Sundin A et al. ENETS Consensus Guidelines Update for Gastroduodenal Neuroendocrine Neoplasms. Neuroendocrinology 2016; 103: 119-124.

24. Pape UF, Niederle B, Costa F et al. ENETS Consensus Guidelines for Neuroendocrine Neoplasms of the Appendix (Excluding Goblet Cell Carcinomas). Neuroendocrinology 2016; 103: 144-152.

25. Niederle B, Pape UF, Costa F et al. ENETS Consensus Guidelines Update for Neuroendocrine Neoplasms of the Jejunum and Ileum. Neuroendocrinology 2016; 103: 125-138.

26. Falconi M, Eriksson B, Kaltsas G et al. ENETS Consensus Guidelines Update for the Management of Patients with Functional Pancreatic Neuroendocrine Tumors and Non-Functional Pancreatic Neuroendocrine Tumors. Neuroendocrinology 2016; 103: 153-171.

27. Ramage JK, De Herder WW, Delle Fave G et al. ENETS Consensus Guidelines Update for Colorectal Neuroendocrine Neoplasms. Neuroendocrinology 2016; 103: 139-143.

28. Reubi JC, Waser B. Concomitant expression of several peptide receptors in neuroendocrine tumours: molecular basis for in vivo multireceptor tumour targeting. Eur J Nucl Med Mol Imaging 2003; 30: 781-793.
29. Hubalewska-Dydejczyk A, Fross-Baron K, Golkowski F, Sowa-Staszczak A, Mikolajczak R, Huszno B. ${ }^{99 m}$ Tc-EDDA/HYNIC-Octreotate in Detection of Atypical Bronchial Carcinoid. Exp Clin Endocrinol Diabetes 2007; 115: 47-49.

30. Garcia-Carbonero R, Sorbye H, Baudin E et al. ENETS Consensus Guidelines for High-Grade Gastroenteropancreatic Neuroendocrine Tumors and Neuroendocrine Carcinomas. Neuroendocrinology 2016; 103: 186-194.

31. Pavel M, O'Toole D, Costa F et al. ENETS Consensus Guidelines Update for the Management of Distant Metastatic Disease of Intestinal, Pancreatic, Bronchial Neuroendocrine Neoplasms (NEN) and NEN of Unknown Primary Site. Neuroendocrinology 2016; 103: 172-185.

32. Haug AR, Cindea-Drimus R, Auernhammer CJ et al. The role of 68Ga-DOTATATE PET/CT in suspected neuroendocrine tumors. J Nucl Med 2012; 53: 1686-1692.

33. Wild D, Wicki A, Mansi R et al. Exendin-4-based radiopharmaceuticals for glucagonlike peptide-1 receptor PET/CT and SPECT/CT. J Nucl Med 2010; 51: 1059-1067.

34. Sowa-Staszczak A, Pach D, Mikołajczak R et al. Glucagon-like peptide-1 receptor imaging with [Lys 40(Ahx-HYNIC-99mTc/EDDA)NH2]-exendin-4 for the detection of insulinoma. Eur. J. Nucl. Med. Mol. Imaging 2013; 38: 1669-1674.

35. Grimes J, Celler A, Birkenfeld B et al. Patient-Specific Radiation Dosimetry of ${ }^{99 m}$ TC-HYNIC-Tyr ${ }^{3}$-Octreotide in Neuroendocrine Tumors. J Nucl Med 2011; 52: 1474-1481.

36. Wild D, Bomanji JB, Benkert $P$ et al. Comparison of ${ }^{68} \mathrm{Ga}-\mathrm{DOTANOC}$ and ${ }^{68} \mathrm{Ga}$-DOTATATE PET/CT within patients with gastroenteropancreatic neuroendocrine tumors. J Nucl Med. 2013; 54: 364-372.

37. Has Simsek D, Kuyumcu S, Turkmen C et al. Can complementary 68GaDOTATATE and 18F-FDG PET/CT establish the missing link between histopathology and therapeutic approach in gastroenteropancreatic neuroendocrine tumors? J Nucl Med. 2014; 55: 1811-1817.

38. Binderup $T$, Knigge $U$, Loft $A$ et al. Functional imaging of neuroendocrine tumors: a head-to-head comparison of somatostatin receptor scintigraphy, 123I-MIBG scintigraphy, and 18F-FDG PET. J Nucl Med 2010; 51: 704-712.

39. Binderup T, Knigge U, Loft A, Kjaer A. 18F-fluorodeoxyglucose positron emission tomography predicts survival of patients with neuroendocrine tumors. Clin Cancer Res 2010; 16: 978-985

40. Ambrosini V, Tomassetti $P$, Castellucci $P$ et al. Comparison between 68Ga-DOTA-NOC and 18F-DOPA PET for the detection of gastro-entero-pancreatic and lung neuro-endocrine tumours. Eur J Nucl Med Mol Imaging 2008; 35: 1431-1438.

41. Haug AR, Auernhammer CJ, Wängler B et al. 68Ga-DOTATATE PET/CT for the early prediction of response to somatostatin receptor-mediated radionuclide therapy in patients with well-differentiated neuroendocrine tumors. J Nucl Med 2010; 51: 1349-1356.

42. Naji M, Zhao C, Welsh SJ et al. 68Ga-DOTA-TATE PET vs. 123I-MIBG in identifying malignant neural crest tumours. Mol Imaging Biol 2011; 13: 769-775.

43. Haug A, Auernhammer CJ, Wängler B et al. Intraindividual comparison of 68Ga-DOTA-TATE and 18F-DOPA PET in patients with well-differentiated metastatic neuroendocrine tumours. Eur J Nucl Med Mol Imaging 2009; 36: 765-770

44. Shan L. ${ }^{18} \mathrm{~F}-$ Fluoroethyl triazole- $\beta$ AG- $\left[(\mathrm{d})-\mathrm{Phe}^{1}-\mathrm{c}\left(\mathrm{Cys}^{2}-\mathrm{Tyr}^{3}-(\mathrm{d})-\mathrm{Trp}^{4}-\mathrm{Lys}^{5}-\right.\right.$ - Thr $\left.^{6}-\mathrm{Cys}^{7}\right) \mathrm{Thr}^{8}$ ]. Molecular Imaging and Contrast Agent Database (MICAD) [Internet]. Bethesda (MD): National Center for Biotechnology Information (US); 2004-2013.

45. Orlefors $\mathrm{H}$, Sundin $\mathrm{A}$, Ahlström $\mathrm{H}$ et al. Positron emission tomography with 5-hydroxytryprophan in neuroendocrine tumors. J Clin Oncol 1998; 16: 2534-2541.

46. Kosowicz J, Mikołajczak R, Czepczynski R, Ziemnicka K, Sowiński J. Two peptide receptor ligands 99mTc-EDDA/HYNIC-Tyr3-Octreotide and 99mTc-EDDA/HYNIC-DGlu-Octagastrin for scintigraphy of medullary thyroid carcinoma." Cancer Biotherapy\&Radiopharmaceuticals 2007; 5: 613. 
47. Maina T, Konijneneberg MW, Kolenc-Peitl P et al. Preclinical pharmacokinetics, biodistribution, radiation dosimetry and toxicity studies of $111 \mathrm{ln}-\mathrm{CP} 04$ required for regulatory approval of a Phase I clinical trial in medullary thyroid carcinoma patients. Eur J Pharm Sci 2016 25; 91: 236-242.

48. Pfeifer A, Knigge $U$, Binderup T et al. 64Cu-DOTATATE PET for Neuroendocrine Tumors: A Prospective Head-to-Head Comparison with 111In-DTPA-Octreotide in 112 Patients. J Nucl Med 2015; 56: 847-854.

49. Hanaoka $\mathrm{H}$, Tominaga $\mathrm{H}$, Yamada $\mathrm{K}$ et al. Evaluation of (64)Cu-labeled DOTA-D-Phe(1)-Tyr (3)-octreotide ((64)Cu-DOTA-TOC) for imaging somatostatin receptor-expressing tumors. Ann Nucl Med 2009; 23: 559-567.
50. Fani M, Mueller A, Tamma ML et al Radiolabeled bicyclic somatostatin-based analogs: a novel class of potential radiotracers for SPECT/PET of neuroendocrine tumors. J Nucl Med 2010; 51: 1771-1779.

51. Reubi JC, Waser B. Triple-peptide receptor targeting in vitro allows detection of all tested gut and bronchial NETs. J Nucl Med 2015; 56: 613-615.

52. Wild D, Fani $M$, Behe $M$ et al. First clinical evidence that imaging with somatostatin receptor antagonists is feasible. J Nucl Med 2011; 52: 1412-1417.

53. Fani M, Braun F, Waser B et al. Unexpected sensitivity of sst2 antagonists to N-terminal radiometal modifications. J Nucl Med 2012; 53: 1481-1489. 